\title{
A Set of Globally Stable N-PID Regulators for Robotic Manipulators
}

\author{
Baishun Liu, Fancai Lin, Bingli Tian \\ Department of Battle \& Command, Academy of Naval Submarine, Qingdao, China \\ E-mail:baishunliu@163.com,lfcai_777@yahoo.com.cn,woshixiaobenmiao@126.com \\ Received August 28, 2009; revised September 28, 2009; accepted October 4, 2009
}

\begin{abstract}
This paper deals with the position control of robot manipulators with uncertain and varying-time payload. Proposed is a set of novel N-PID regulators consisting of a linear combination of the proportional control mode, derivative control mode, nonlinear control mode shaped by a nonlinear function of position errors, linear integral control mode driven by differential feedback, and nonlinear integral control mode driven by a nonlinear function of position errors. By using Lyapunov's direct method and LaSalle's invariance principle, the simple explicit conditions on the regulator gains to ensure global asymptotic stability are provided. The theoretical analysis and simulation results show that: an attractive feature of our scheme is that N-PID regulators with asymptotic stable integral actions have the faster convergence, better flexibility and stronger robustness with respect to uncertain and varying-time payload, and then the optimum response can be achieved by a set of control parameters in the whole control domain, even under the case that the payload is changed abruptly.
\end{abstract}

Keywords: Manipulators, Robot Control, PID Control, N-PID Control, Global Stability

\section{Introduction}

It is well known that PID controllers can effectively deal with nonlinearity and uncertainties of dynamics, and asymptotic stability is achieved accordingly [1-3]. Hence, most robots employed in industrial operations are controlled through PID controllers that introduce integral action by integrating the error. It is well known that integral-action controllers with this class of integrator often suffer a serious loss of performance due to integrator windup, which occurs when the actuators in the control loop saturate. Actuator saturation not only deteriorates the control performance, causing large overshoot and long settling time, but also can lead to instability, since the feedback loop is broken for such saturation. To avoid this drawback, various PID-like controllers have been proposed to improve the transient performance. For example, PID controllers consisting of a saturated-P, and differential feedback plus a PI controller driven by a bounded nonlinear function of position errors [4], a linear PD feedback plus an integral action of a nonlinear function of position errors [5], a linear derivative feedback plus a PI term driven by a nonlinear function of position errors [1], a linear PD feedback plus double integral action driven by the positions error and the filtered position [6], a linear PD feedback plus an integral action driven by PD controller [7], and a linear PD feedback plus an integral action driven by NP-D controller [8], are presented recently.

In this paper, we propose a set of new global position controllers for robots which do not include their dynamics in the control laws. Motivated by the idea that is introducing the nonlinear action of the position errors into PD-NI controller [5] and modifying the nonlinear integral action via injection of the required damping so that the transient performance of the closed-loop system may be improved, we develop a set of new N-PID-like regulators consisting of a linear combination of the proportional control mode, derivative control mode, nonlinear control mode shaped by a nonlinear function of position errors, linear integral control mode driven by differential feedback, and nonlinear integral control mode driven by a nonlinear function of position errors. The simple explicit conditions on the regulator gains to ensure global asymptotic stability are provided.

Throughout this paper, we use the notation $\lambda_{m}(A)$ and $\lambda_{M}(A)$ to indicate the smallest and largest eigenvalues, respectively, of a symmetric positive define bounded matrix $A(x)$, for any $x \in R^{n}$. The norm of vector $x$ is defined as $\|x\|=\sqrt{x^{T} x}$, and that of matrix $A$ 
is defined as the corresponding induced norm $\|A\|=\sqrt{\lambda_{M}\left(A^{T} A\right)}$.

The remainder of the paper is organized as follows. Section 2 summarizes the robot model and its main properties. Our main results are presented in Sections 3 and 4, where we briefly review some known PID-like control laws and present a set of new N-PID-like control laws, and then provide the conditions on the controller gains to ensure global asymptotic stability, respectively. Simulation examples are given in Section 5. Conclusions are presented in Section 6.

\section{Problem Formulation}

The dynamic system of an $n$-link rigid robot manipulator system [1] can be written as:

$$
M(q) \ddot{q}+C(q, \dot{q}) \dot{q}+D \dot{q}+g(q)=u
$$

where $q$ is the $n \times 1$ vector of joint positions, $u$ is the $n \times 1$ vector of applied joint torques, $M(q)$ is the $n \times n$ symmetric positive define inertial matrix, $C(q, \dot{q}) \dot{q}$ is the $n \times 1$ vector of the Coriolis and centrifugal torques, $D$ is the $n \times n$ positive define diagonal friction matrix, and $g(q)$ is the $n \times 1$ vector of gravitational torques obtained as the gradient of the robot potential energy $U(q)$ due to gravity.

A list of properties [1] of the robot dynamic model (1) is recalled as follows:

$$
\begin{gathered}
0<\lambda_{m}(M) \leq\|M(q)\| \leq \lambda_{M}(M) \\
\zeta^{T}(\dot{M}(q)-2 C(q, \dot{q})) \zeta=0 \quad \forall \zeta \in R^{n} \\
0<C_{m}\|\dot{q}\|^{2} \leq\|C(q, \dot{q}) \dot{q}\| \leq C_{M}\|\dot{q}\|^{2} \quad \forall q, \dot{q} \in R^{n}
\end{gathered}
$$

where $C_{m}$ and $C_{M}$ are all positive constants.

For the purpose of this paper, it is convenient to introduce the following definition and properties [5].

Definition 1: $F(\alpha, \beta, x)$ with $1 \geq \alpha>0, \beta>0$, and $x \in R^{n}$ denotes the set of all continuous differential increasing functions,

$$
\begin{gathered}
f(x)=\left[f\left(x_{1}\right), \cdots, f\left(x_{n}\right)\right]^{T} \text { such that } \\
\qquad \begin{array}{c}
|x| \geq|f(x)| \geq \alpha|x| \quad \forall x \in R:|x|<\beta \\
\beta \geq|f(x)| \geq \alpha \beta \quad \forall x \in R:|x| \geq \beta \\
1 \geq(d / d x) f(x) \geq 0
\end{array}
\end{gathered}
$$

where $|\bullet|$ stands for the absolute value.

Figure 1 depicts the region allowed for functions belonging to $\operatorname{set} F(\alpha, \beta, x)$. For instance, the tangent hyperbolic function belongs to set $F(\tanh (1), 1, x)$ and the Arimoto sine function, whose entries are given as follows:

$$
f(x)= \begin{cases}1 & \text { if } x \geq \pi / 2 \\ \sin (x) & \text { if }|x|<\pi / 2 \\ -1 & \text { if } x \leq-\pi / 2\end{cases}
$$

which belongs to set $F(\sin (1), 1, x)$.

The important properties of function $f(x)$ belonging to set $F(\alpha, \beta, x)$ are now established.

The function $x^{T} f(x)$ satisfies for all $x \in R^{n}$,

$$
x^{T} f(x) \geq 0
$$

The Euclidean norm of $f(x)$ satisfies for all $x \in R^{n}$,

$$
\begin{aligned}
& \|f(x)\|^{2} \leq\|x\|^{2} \\
& \|f(x)\| \leq \sqrt{n} \beta
\end{aligned}
$$

Throughout this paper, we use the notation $\Delta q=q-q_{d}$, to indicate the position errors, $q_{d}$ is the desired joint position, which is assumed to be constant; $K_{P}, K_{D}$, $K_{S}, K_{P f}, K_{I}, K_{I P}$ and $K_{I D}$ are all positive define diagonal $n \times n$ matrices.

\section{N-PID-Like Control Laws}

\subsection{PID-Like Control Law Review}

To put our contribution in perspective, we will briefly review some known PID-like control laws, as follows:

1) Semiglobally stable PID control law [1],

$$
u=-K_{P} \Delta q-K_{D} \dot{q}-K_{I} \int_{0}^{t} \Delta q(\tau) d \tau
$$

2) Globally stable PD-NI control law [1],

$$
u=-K_{P} \Delta q-K_{D} \dot{q}-K_{I} \int_{0}^{t} \tanh (\Delta q(\tau)) d \tau .
$$

where $\tanh (\bullet)$ is the hyperbolic tangent vector function.

3) Globally stable PD-NPI control law [1],

$$
u=-K_{P} \Delta q-K_{D} \dot{q}-K_{S} s(\Delta q)-K_{I} \int_{0}^{t} s(\Delta q(\tau)) d \tau .
$$

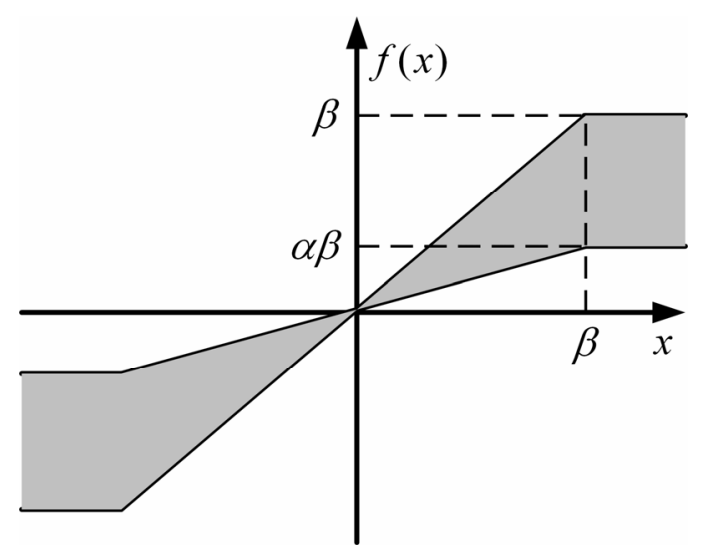

Figure 1. $F(\alpha, \beta, x)$ functions. 
where $s(\bullet)$ is the differential function of a class of approximate potential energy function.

4) Semiglobally stable $\mathrm{PI}^{2} \mathrm{D}$ control law [6],

$$
u=-K_{P} \Delta q-K_{D} \dot{q}-K_{I} \int_{0}^{t}[\Delta q(\tau)+\Delta \dot{q}(\tau)] d \tau .
$$

5) Semiglobally stable PD-I ${ }^{\mathrm{PD}}$ control law [7],

$$
u=-K_{P} \Delta q-K_{D} \dot{q}-\int_{0}^{t}\left[K_{I P} \Delta q(\tau)+K_{I D} \Delta \dot{q}(\tau)\right] d \tau .
$$

6) Globally stable PD-I ${ }^{\text {NP-D }}$ control law [8],

$$
\begin{aligned}
& u=-K_{P} \Delta q-K_{D} \dot{q} \\
& -\int_{0}^{t}\left[K_{I P} \tanh (\Delta q(\tau))+K_{I D} \Delta \dot{q}(\tau)\right] d \tau
\end{aligned}
$$

7) Globally stable SP-D-NPI control law [4],

$$
\begin{aligned}
& u=-K_{P} \operatorname{sat}(\Delta q)-K_{D} \dot{q}-K_{P f} f_{b}(\Delta q) \\
& -K_{I} \int_{0}^{t} f_{b}(\Delta q(\tau)) d \tau
\end{aligned} .
$$

where $\operatorname{sat}(\bullet)$ is the normal saturated function and $f_{b}(\bullet)$ is a bounded nonlinear function.

8) Globally stable PD-NI control law [5],

$$
u=-K_{P} \Delta q-K_{D} \dot{q}-K_{I} \int_{0}^{t} f(\Delta q(\tau)) d \tau
$$

where $f(\bullet)$ is a continuous differential increasing bounded functions shown in Figure 1.

Most of the present industrial robots are controlled through PID-like controllers above. Although these controllers have been shown in practice to be effective for position control of robot manipulators, unfortunately most of them often suffer a serious loss of performance, that is, causes large overshoot and long settling time due to unlimited integral action.

Based on the above fact, we get intuitively an idea that the transient performance of the closed-loop system may be improved if the nonlinear action of position errors is introduced into the control law (10) and the damping is injected into its integrator. Following this idea, a set of novel N-PID-like regulators is developed in the next subsection.

\subsection{Our N-PID Control Laws}

The new nonlinear PID control laws are proposed as follows,

$$
\begin{aligned}
& u=-K_{P} \Delta q-K_{D} \dot{q}-K_{P f} f(\Delta q) \\
& -\int_{0}^{t}\left[K_{I P} f(\Delta q(\tau))+K_{I D} \Delta \dot{q}(\tau)\right] d \tau
\end{aligned}
$$

It is worthy to note that the control law above is consisting of a linear combination of the proportional control mode, derivative control mode, nonlinear control mode, linear integral control mode and nonlinear integral control mode. Hence, based on the five control modes above, five differential N-PID-like control laws with the same stability as the control law (11) can be derived, as follows:

$$
\begin{aligned}
& u=-K_{P} \Delta q-K_{D} \dot{q}-\int_{0}^{t}\left[K_{I P} f(\Delta q(\tau))+K_{I D} \Delta \dot{q}(\tau)\right] d \tau \\
& u=-K_{D} \dot{q}-K_{P f} f(\Delta q) \\
& -\int_{0}^{t}\left[K_{I P} f(\Delta q(\tau))+K_{I D} \Delta \dot{q}(\tau)\right] d \tau \\
& u=-K_{D} \dot{q}-\int_{0}^{t}\left[K_{I P} f(\Delta q(\tau))+K_{I D} \Delta \dot{q}(\tau)\right] d \tau \\
& u=-K_{P} \Delta q-K_{D} \dot{q}-K_{I P} \int_{0}^{t} f(\Delta q(\tau)) d \tau \\
& u=-K_{P} \Delta q-K_{D} \dot{q}-K_{P f}(\Delta q)-K_{I P} \int_{0}^{t} f(\Delta q(\tau)) d \tau
\end{aligned}
$$

Discussion 1: It is obvious that the control law (11) can be simplified to other PID-like control laws such as P-NI control, NPI control, PD-I ${ }^{\mathrm{NP}-\mathrm{D}}$ control [8], and so on. Moreover, the control law (15) is the same as the one (10) reported by [5]. This shows that the control law (11) is a natural extension of them and implies that its application is not limited in the robots, too.

Discussion 2: Notice that the integral actions in control laws (10) and (11) can be rewritten as $\dot{\sigma}=-K_{I} f(\Delta q)$ and $\dot{\sigma}=-K_{I P} f(\Delta q)-K_{I D} \Delta \dot{q}$, respectively. From this, it is easy to see that the latter has the same stability as the one presented by $[7,8]$, that is, they are all asymptotically stable but the former has the same stability as the classical integral action, $\dot{\sigma}=-K_{I} \Delta q$, that is, they are all only stable. This means that the controller (11)-(14) should have faster convergence, better flexibility than the one (10), and then the controller (11)-(14) can yield higher performance of control, too. Moreover, integrator windup can be avoided by choosing suitable parameters $K_{I P}$ and $K_{I D}$.

Discussion 3: Compared to the classical PID control and PD-NI control [5], the following observations can be made during the control process: when $K_{I P} f(\Delta q)+K_{I D} \Delta \dot{q}=0$, the integral action remains constant; if the integral action is large, $\Delta \dot{q}$ increases, and then the integral action instantly decreases, vice versa. However, the integral action produced by the classical or nonlinear integrator [5] always increases as long as the error does not cross over zero, only when the error crosses over zero, the integral action will start to decrease. This shows that the control laws with the asymptotically stable integrator should have the faster convergence and better flexibility, and then can yield higher transient performance, once again.

\section{Stability Analysis}

For analyzing the stability of the closed-loop system, it is convenient to introduce the following notation.

$$
\text { Defining } z(t)=\int_{0}^{t} f(\Delta q(\tau)) d \tau+K_{I P}^{-1}\left[g\left(q_{d}\right)-K_{I D} \Delta q(0)\right] \text {, }
$$


and then the control law (11) can be rewritten as,

$$
\begin{aligned}
& u=-\left(K_{P}+K_{I D}\right) \Delta q-K_{D} \dot{q} \\
& -K_{P f} f(\Delta q)-K_{I P} z+g\left(q_{d}\right)
\end{aligned}
$$

The closed-loop system dynamics is obtained by substituting the control action $u$ from (17) into the robot dynamic model (1),

$$
\begin{aligned}
& M(q) \ddot{q}+C(q, \dot{q}) \dot{q}+\left(K_{D}+D\right) \dot{q}+g(q)-g\left(q_{d}\right) \\
& +\left(K_{P}+K_{I D}\right) \Delta q+K_{I P} z+K_{P f} f(\Delta q)=0
\end{aligned}
$$

From the closed-loop system dynamics above, it is easy to see that the origin $\left(\Delta q^{T}, \dot{q}^{T}, z^{T}\right)^{T}=0 \in R^{3 n}$ is the unique equilibrium point.

Now, the objective is to provide conditions on the controller gains $K_{P}, K_{D}, K_{P f}, K_{I P}$ and $K_{I D}$ ensuring global asymptotic stability of the unique equilibrium point. This is established in the following.

Theorem: Consider the robot dynamics (1) together with control law (11). There exists positive constant $a$ small enough, and choose the gain matrices $K_{P}, K_{D}$, $K_{P f}, K_{I P}$ and $K_{I D}$ such that

$$
\begin{aligned}
& K_{P}+K_{I D}>K_{I P}+4 \lambda_{M}(M) I \\
& U(q)-U\left(q_{d}\right)-\Delta q^{T} g\left(q_{d}\right) \\
& +\frac{1}{4} \Delta q^{T}\left(K_{P}+K_{I D}-K_{I P}\right) \Delta q \geq a\|\Delta q\|^{2} \\
& f^{T}(\Delta q)\left[g(q)-g\left(q_{d}\right)\right]+f^{T}(\Delta q) K_{P f} f(\Delta q) \\
+ & f^{T}(\Delta q)\left(K_{P}+K_{I D}-K_{I P}\right) \Delta q \\
\geq & a\|f(\Delta q)\|^{2} \\
& K_{D}+D>\left(\lambda_{M}(M)+C_{M} \sqrt{n} \beta\right) I
\end{aligned}
$$

hold, and then the closed-loop system (18) is globally asymptotically stable, i.e. $\lim _{t \rightarrow \infty} \Delta q=0$.

Proof: To carry out the stability analysis, we consider the following Lyapunov function candidate:

$$
\begin{aligned}
& V=\frac{1}{2} \dot{q}^{T} M(q) \dot{q}+\frac{1}{2}(z+\Delta q)^{T} K_{I P}(z+\Delta q) \\
& +U(q)-U\left(q_{d}\right)-\Delta q^{T} g\left(q_{d}\right)+f^{T}(\Delta q) M(q) \dot{q} \\
& +\frac{1}{2} \Delta q^{T}\left(K_{P}+K_{I D}-K_{I P}\right) \Delta q \\
& +\sum_{i=1}^{n} \int_{0}^{\Delta q_{i}} f^{T}\left(x_{i}\right)\left(K_{P f i}+K_{D i}+D_{i}\right) d x_{i}
\end{aligned}
$$

where $x_{i}=\Delta q_{i} ; K_{P f i}, K_{D i}$ and $D_{i}$ are the diagonal element of the matrices $K_{P f}, K_{D}$ and $D$, respectively.

1) Positive definition of Lyapunov function candidate.

Now, considering the following inequality, and using (8), we have,

$$
\begin{aligned}
& \frac{1}{4} \dot{q}^{T} M(q) \dot{q}+\frac{1}{4} \Delta q^{T}\left(K_{P}+K_{I D}-K_{I P}\right) \Delta q \\
& +f^{T}(\Delta q) M(q) \dot{q} \\
& =\frac{1}{4}[\dot{q}+2 f(\Delta q)]^{T} M(q)[\dot{q}+2 f(\Delta q)] \\
& -f^{T}(\Delta q) M(q) f(\Delta q) \\
& +\frac{1}{4} \Delta q^{T}\left(K_{P}+K_{I D}-K_{I P}\right) \Delta q \\
& \geq \frac{1}{4} f^{T}(\Delta q)\left(K_{P}+K_{I D}-K_{I P}-4 \lambda_{M}(M) I\right) f(\Delta q)
\end{aligned}
$$

Substituting (20) and (24) into (23), and using (7) and (19), for any $\left(\Delta q^{T}, \dot{q}^{T}, z^{T}\right)^{T} \neq 0$, we obtain,

$$
\begin{aligned}
& V=\frac{1}{4} \dot{q}^{T} M(q) \dot{q}+a\|\Delta q\|^{2} \\
& +\sum_{i=1}^{n} \int_{0}^{\Delta q_{i}} f^{T}\left(x_{i}\right)\left(K_{P f i}+K_{D i}+D_{i}\right) d x_{i} \\
& +\frac{1}{2}(z+\Delta q)^{T} K_{I P}(z+\Delta q)>0
\end{aligned}
$$

This shows that the Lyapunov function candidate (23) is positive define.

2) Time derivative of Lyapunov function candidate.

The time derivative of Lyapunov function candidate (23) along the trajectories of the closed-loop system (18) is,

$$
\begin{aligned}
& \dot{V}=\dot{q}^{T} M(q) \ddot{q}+\frac{1}{2} \dot{q}^{T} \dot{M}(q) \dot{q}+(\dot{f}(\Delta q) \Delta \dot{q})^{T} M(q) \dot{q} \\
& +f^{T}(\Delta q) \dot{M}(q) \dot{q}+f^{T}(\Delta q) M(q) \ddot{q} \\
& +\dot{q}^{T} g(q)-\Delta \dot{q}^{T} g\left(q_{d}\right)+\Delta \dot{q}^{T}\left(K_{P}+K_{I D}-K_{I P}\right) \Delta q \\
& +f^{T}(\Delta q)\left(K_{P f}+K_{D}+D\right) \Delta \dot{q}+(\dot{z}+\Delta \dot{q})^{T} K_{I P}(z+\Delta q)
\end{aligned}
$$

Substituting $\dot{z}=f(\Delta q)$, and $M(q) \ddot{q}$ from (18) into (26), and using (3), we have,

$$
\begin{aligned}
& \dot{V}=-\dot{q}^{T}\left(K_{D}+D\right) \dot{q}+(\dot{f}(\Delta q) \Delta \dot{q})^{T} M(q) \dot{q} \\
& +f^{T}(\Delta q) C(q, \dot{q}) \dot{q}-f^{T}(\Delta q)\left[g(q)-g\left(q_{d}\right)\right] \\
& -f^{T}(\Delta q)\left(K_{P}+K_{I D}-K_{I P}\right) \Delta q \\
& -f^{T}(\Delta q) K_{P f} f(\Delta q)
\end{aligned}
$$

Now, using (2) and (5), we get,

$$
(\dot{f}(\Delta q) \Delta \dot{q})^{T} M(q) \dot{q} \leq \lambda_{M}(M)\|\dot{q}\|^{2}
$$

By using (4) and (9), we obtain,

$$
f^{T}(\Delta q) C^{T}(q, \dot{q}) \dot{q} \leq C_{M} \sqrt{n} \beta\|\dot{q}\|^{2}
$$

Incorporating (21), (28) and (29) into (27), we obtain,

$$
\begin{aligned}
& \dot{V} \leq-\dot{q}^{T}\left(K_{D}+D-\lambda_{M}(M) I-C_{M} \sqrt{n} \beta I\right) \dot{q} \\
& -a\|f(\Delta q)\|^{2}
\end{aligned}
$$

From (22), (30) and $a>0$, we can conclude $\dot{V} \leq 0$. 
Using the fact that the Lyapunov function candidate (23) is a positive define function and its time derivative is a negative semi define function, we conclude that the equilibrium point of the closed-loop system (18) is stable. In fact, $\dot{V}=0$ means $\Delta q=0$ and $\dot{q}=0$. By invoking the LaSalle's invariance principle, it is easy to know that the equilibrium point $\left(\Delta q^{T}, \dot{q}^{T}\right)^{T}=0$ is globally asymptotically stable, i.e., $\lim _{t \rightarrow \infty} \Delta q=0$.

Remark 1: For the control laws (12)-(16), the globally asymptotically stable results can be derived under some similar sufficient conditions presented by (19)-(22). The proof can follow the similar argument and procedure. It is omitted because of the limited space.

Discussion 4: From the proof procedure above, it is easy to see that: 1 ) if we chooses gain matrix, $K_{I D}$ large enough, the linear term, $K_{P} \Delta q$, and the nonlinear term, $K_{P f} f(\Delta q)$, in the control law (11) is not necessary for guaranteeing global asymptotic stability of the closedloop system and this means that the global asymptotic stability can still be ensured by the simplified form of the control law $(11) ; 2)$ on the other hand, with the linear term, $K_{P} \Delta q$, and the nonlinear term, $K_{P f} f(\Delta q)$, it is obvious that the condition (19)-(21) is more easily satisfied and this results in that the control engineers have more freedom to choose the controller parameters, and then make them more easily tune a high performance controller.

\section{Simulations}

To illustrate the effect of the controller given in this paper, two-link manipulators shown in Figure 2 are considered. The dynamics (1) is of the following form [9]

$$
\left\{\begin{array}{l}
M_{11} \ddot{q}_{1}+M_{12} \ddot{q}_{2}+F_{11} \dot{q}_{2}^{2}+2 F_{12} \dot{q}_{1} \dot{q}_{2}+G_{1}=u_{1} \\
M_{12} \ddot{q}_{1}+M_{22} \ddot{q}_{2}+F_{11} \dot{q}_{1}^{2}+2 F_{12} \dot{q}_{1} \dot{q}_{2}+G_{2}=u_{2}
\end{array}\right.
$$

where: $M_{11}=\left(m_{1}+m_{2}\right) l_{1}^{2}+m_{2} l_{2}^{2}+2 m_{2} l_{1} l_{2} \cos \left(q_{2}\right)$,

$$
\begin{aligned}
& M_{22}=m_{2} l_{2}^{2}, \quad M_{12}=m_{2} l_{2}^{2}+m_{2} l_{1} l_{2} \cos \left(q_{2}\right), \\
& F_{11}=F_{12}=-m_{2} l_{1} l_{2} \sin \left(q_{2}\right), \\
& G_{1}=\left(m_{1}+m_{2}\right) g l_{1} \sin \left(q_{1}\right)+m_{2} g l_{2} \sin \left(q_{1}+q_{2}\right) \\
& G_{2}=m_{2} g l_{2} \sin \left(q_{1}+q_{2}\right) .
\end{aligned}
$$

The normal parameter values of the system are selected as: $m_{1}=m_{2}=1 \mathrm{~kg}, l_{1}=l_{2}=1 \mathrm{~m}, g=10 \mathrm{~m} / \mathrm{s}^{2}$.

The desired (set point) positions are chosen as:

when $t<10 s, q_{d 1}=1, q_{d 2}=-1$;

when $10 s \leq t<20 s, q_{d 1}=3, q_{d 2}=2$;

when $t \geq 20 s, q_{d 1}=q_{d 2}=0$.

The simulation is implemented by using the control laws (11) and (14), respectively. In simulation, Arimoto

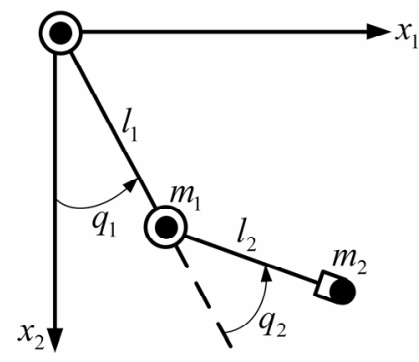

Figure 2. The two-link robot manipulators.

sine function (6) is used as the nonlinear function of the control laws.

The gain matrices of the control law (11) are selected as:

$$
\begin{aligned}
& K_{P}=\operatorname{diag}(310,310), \quad K_{D}=\operatorname{diag}(150,150), \\
& K_{I P}=\operatorname{diag}(500,500), \quad K_{I D}=\operatorname{diag}(200,200),
\end{aligned}
$$

and $K_{P f}=\operatorname{diag}(100,100)$.

The gain matrices of the control law (14) are given as:

$$
K_{D}=\operatorname{diag}(60,30), K_{I P}=\operatorname{diag}(1000,500) \text {, }
$$

and $K_{I D}=\operatorname{diag}(1250,600)$.

The simulations with sampling period of $2 \mathrm{~ms}$ are implemented. Figures 3 and 5 present the response of the robot manipulators under the normal parameters. Figures 4 and 6 are the simulation results under the case that the mass $m_{2}=1 \mathrm{~kg}$ is substituted for $m_{2}=3 \mathrm{~kg}$ when $10 s \leq t<20 s$, corresponding to moving payload of $2 \mathrm{~kg}$.

From the simulation results, it is easy to see that:

1) The optimum response can all be achieved, respectively, by the control laws (11) and (14) with a set of control parameters in the whole domain of interest, even under the case that the payload is changed abruptly;

2) These tow controllers used in simulation all have the faster convergence, better flexibility and stronger robustness with respect to uncertain payload, which means that the controllers (12) and (13) should have the same high performance of control as the controllers (11) and (14) because they all employ the same integrators;

3 ) Comparing the Figures 3 and 5, the controller (11) is easier to achieve the control of high speed and high performance than the controller (14) because the former has two freedom parameters.

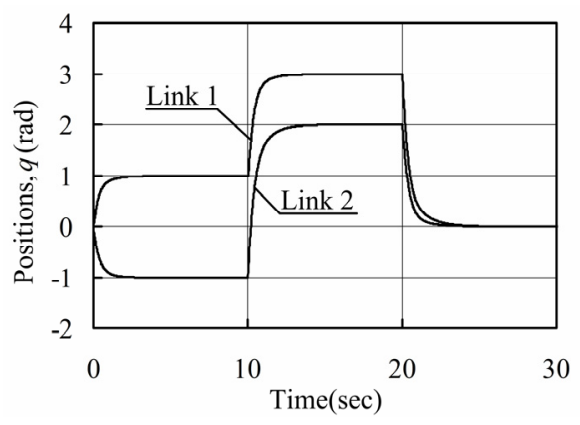

Figure 3. Under normal parameters, the simulation results with controller (11). 


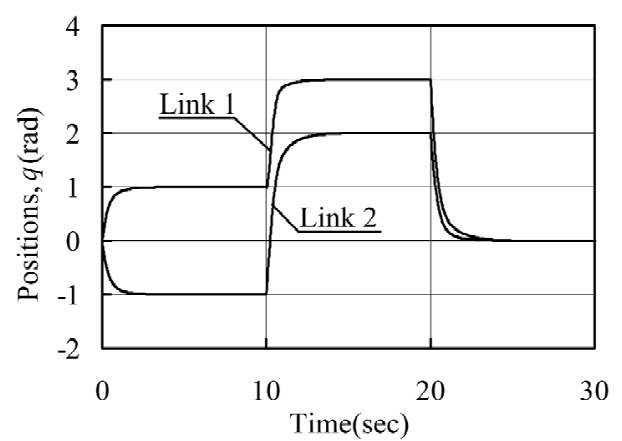

Figure 4. Under perturbed parameters, corresponding to moving payload, the simulation results with controller (11).

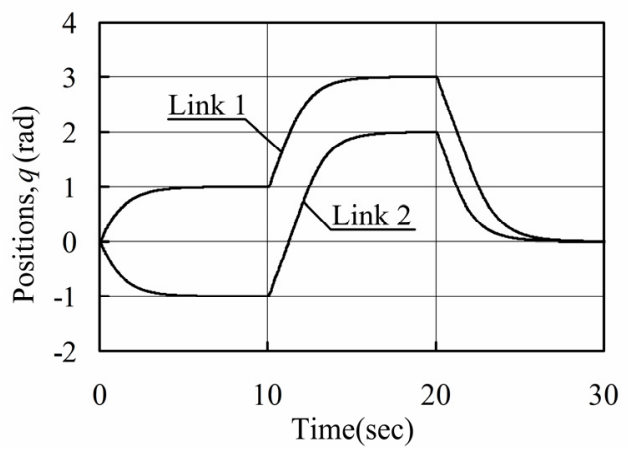

Figure 5. Under normal parameters, the simulation results with controller (14).

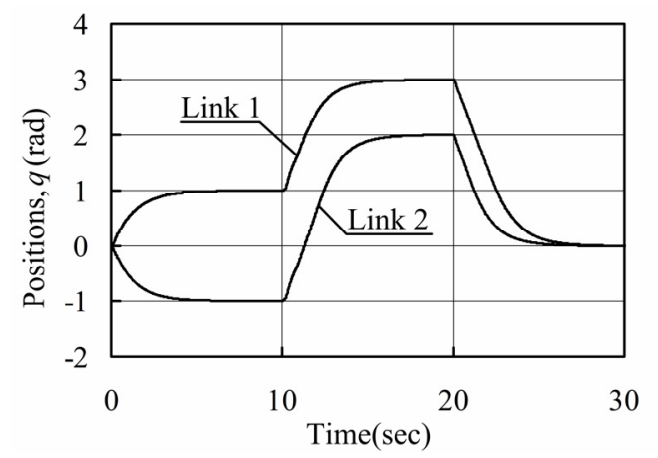

Figure 6. Under perturbed parameters, corresponding to moving payload, the simulation results with controller (14).

\section{Conclusions}

In this paper, we have presented a set of solution to the problem of set point regulation for rigid robots without exact knowledge of the payload. An attractive feature of our scheme is that the control laws with the asymptotically stable integrators have the faster convergence, better flexibility and stronger robustness with respect to uncertain and varying-time payload, and then the optimum response can be achieved. The explicit conditions on the regulator gains to ensure global asymptotic stability of the overall closed-loop system are given in terms of some information exacted from the robot dynamics. Our findings have been corroborated numerically on a two DOF vertical robot manipulators.

\section{References}

[1] Y. X. Su, Nonlinear Control Theory for Robot Manipulators, Science Publishing, Beijing, 2008.

[2] J. Alvarez-Ramirez, I. Gervantes, and R. Kelly, "PID regulation of robot manipulators: Stability and performance," System \& Control Letters, Vol. 41, No. 2, pp. 73-83, April 2000.

[3] A. A. Pervozvanski and L. B. Freidovich, "Robust stability of robotic Manipulators by PID controllers," Dynamics and Control, Vol. 9, No. 3, pp. 203-222, September 1999.

[4] S. Arimoto, "A class of quasi-natural potentials and Hyper-stable PID servo-loops for nonlinear robotic system," Transactions of the Society of Instrument and Control Engineers, Vol. 30, No. 9, pp. 1005-1012, September 1994.

[5] R. Kelly, "Global positioning of robotic manipulators via PD control plus a class of nonlinear integral actions," IEEE Transactions on Automatic Control, Vol. 43, No. 7, pp. 934-938, July 1998.

[6] R. Ortega, A. Loria, and R. Kelly, "A semiglobally stable output feedback $\mathrm{PI}^{2} \mathrm{D}$ regulator for robot manipulators," IEEE Transactions on Automatic Control, Vol. 40, No. 8, pp. 1432-1436, August 1995.

[7] B. S. Liu and F. C. Lin, "A semiglobally stable PD-I ${ }^{\mathrm{PD}}$ regulator for robot manipulators," to be published in 2009 International Conference on Measuring Technology and Mechatronics Automation proceeding.

[8] B. S. Liu and B. L. Tian, "A globally stable PD-I ${ }^{\text {NP-D }}$ regulator for robot manipulators," to be published in 2009 International Conference on Information Technology and Computer Science proceedings.

[9] S. J. Yu, X. D. Qi, and J. H. Wu, Iterative Learning Control Theory \& Application, Machine Publishing, Beijing, 2005. 